\title{
DÉCHARGES ÉLECTRIQUES DANS LE VIDE : IMPACT DE MICROPARTICULES SPHÉRIQUES DE FER DE LA TAILLE DU MICRON SUR DES ÉLECTRODES POLIES MÉCANIQUEMENT
}

\section{I : EFFETS MÉCANIQUES DU CHOC}

\author{
C. TEXIER
}

Laboratoire de Physique Expérimentale, Institut de Physique de l'Université de Nantes, Rue de la Houssinière, 44072 Nantes Cedex, France

(Reçu le ler décembre 1976, révisé le 7 juillet 1977, révisé le 9 octobre 1977, accepté le 17 octobre 1977)

Résumé. - On a étudié l'impact de microparticules accélérées électrostatiquement (taille : quelques microns, vitesse : de 20 à $200 \mathrm{~m} / \mathrm{s}$ ) sur une cible métallique $(\mathrm{Al}, \mathrm{Cu}, \mathrm{Ni}, \mathrm{Au}$ ) dans un vide industriel $\left(10^{-5}\right.$ torr) et sous incidence quasi normale.

On montre que le comportement des microparticules est identique à celui d'une bille macroscopique indéformable tombant sur un plan : choc inélastique, diminution du coefficient de restitution $e$ quand la vitesse incidente augmente, indépendance de $e$ vis-à-vis du rayon de la microparticule. L'interprétation théorique de Tabor du choc d'une bille macroscopique reste valable tant qualitativement que quantitativement à condition d'utiliser des formules rigoureuses pour le volume de l'empreinte. Les formules générales devenant alors inextricables, on en a effectué une résolution numérique. On en a déduit la variation du diamètre

et de la profondeur

$$
\begin{array}{ll}
D=C_{1} \times R \times v_{1} & \left(\alpha_{1} \simeq 0,5\right) \\
h=C_{2} \times R \times v_{1}^{\alpha_{2}} & \left(\alpha_{2} \simeq 1,1\right)
\end{array}
$$

de l'empreinte sur la cible en fonction du rayon $R$ et de la vitesse incidente $v_{1}$ de la microparticule, ainsi que la proportionnalité du volume de l'empreinte et de l'énergie cinétique de la microparticule incidente. La valeur trouvée de la limite d'élasticité est supérieure à la valeur macroscopique mesurée par les méthodes de traction ou de compression (environ 4 fois pour l'aluminium). Ces résultats sont en accord avec ceux de Rudolph qui a étudié le même phénomène pour des vitesses plus élevées $(500 \mathrm{~m} / \mathrm{s}-10 \mathrm{~km} / \mathrm{s})$.

Abstract. - We have studied the impact of electrostatically accelerated microparticles (size : a few $\mu \mathrm{m}$, velocity from 20 to $200 \mathrm{~m} / \mathrm{s}$ ) on a metal ( $\mathrm{Al}, \mathrm{Cu}, \mathrm{Ni}, \mathrm{Au}$ ) target in an industrial vacuum $\left(10^{-5}\right.$ torr) under quasi-normal incidence.

We show that microparticles behave identically like macroscopic balls falling on a plane : semi-elastic impact, decrease in the coefficient of restitution $e$ with increasing velocity, $e$ independent of microparticle radius. Tabor's theoretical interpretation of the impact of a macroscopic sphere remains both qualitatively and quantitatively valid provided that exact formulas are used for the volume of indentation. Then, equations cannot be solved and a numerical computation has been used. The variation of the indentation diameter

and depth

$$
\begin{array}{ll}
D=C_{1} \times R \times v_{1}{ }^{1} & \left(\alpha_{1} \simeq 0.5\right) \\
h=C_{2} \times R \times v_{1}^{\alpha_{2}} & \left(\alpha_{2}=1.1\right)
\end{array}
$$

on the target with the microparticle radius $R$ and the impact velocity $v_{1}$ together with the proportionality of the indentation volume to the kinetic energy of the impinging microparticle are deduced from these data. The value of the onset of permanent elastic deformation is larger (4 times larger with $\mathrm{Al}$ ) than the macroscopic value from tensile and compressive strain measurements. The coefficient of restitution is independent of the radius. These results agree with Rudolph's, that studied the same phenomenon for larger velocities $(500 \mathrm{~m} / \mathrm{s}-10 \mathrm{~km} / \mathrm{s})$.

1. Introduction. - Dans le cas des petites distances interélectrodes (inférieures au $\mathrm{cm}$ ), l'amorçage de la décharge électrique dans le vide est attribué à une émission de champ due à la présence sur les électrodes de micropointes présentant un fort coefficient d'amplification du champ électrique (Maitland [1], Alpert 
et al. [2], Brodie [3], Boyle et al. [4]...). Pour les distances interélectrodes plus élevées, le champ électrique de claquage étant trop faible pour produire une émission électronique notable [5], l'amorçage de la décharge est attribué à la présence sur les électrodes de microparticules de la taille du micron et plus. Si dès 1952 Cranberg [6] a, le premier, postulé l'existence de ces microparticules, leur présence n'a été mise en évidence expérimentalement que récemment, soit par une méthode statique (Menon et Srivastava [7], Theophilus et al. [8]), soit par une méthode dynamique (Texier [9]). On trouve dans la littérature de nombreuses hypothèses sur le mécanisme qui conduit de l'impact sur une électrode d'une microparticule arrachée sous l'action des forces électrostatiques à l'intense ionisation de l'espace interélectrode que caractérise la décharge disruptive (Cranberg [6], Slivkov [10], Rohrbach [11], Chatterton et al. [12], Davies et Biondi [13]). Cependant, toutes postulent la présence d'un seuil d'énergie fonction du mécanisme proposé. Latham et Braun [14] ont émis l'hypothèse que ce seuil était atteint après une série de rebondissements sur les deux électrodes, du moins dans le cas d'électrodes planes et parallèles.

Boulloud [15] a montré, à partir de considérations sur les forces d'adhésion, que la taille des microparticules susceptibles de provoquer une décharge est de l'ordre du micron et leur vitesse de quelques centaines de m/s. Ces ordres de grandeur sont en accord avec nos observations de microparticules réelles en vol [9]. Nous avons étudié ici l'impact sur une cible de microparticules de fer carbonyle de quelques microns accélérées électrostatiquement à des vitesses de 20 à $200 \mathrm{~m} / \mathrm{s}$. La cible était soumise à un champ électrique nul ou faible afin de dissocier les effets mécaniques et électriques du choc. Dans cet article, nous ne considérerons que ses aspects mécaniques: variation du coefficient de restitution avec la vitesse incidente, forme, volume, diamètre et profondeur de l'empreinte laissée sur la cible. Cette étude expérimentale avait déjà été effectuée par Rudolph $[16,17]$ dans le cas de vitesses plus élevées $(500 \mathrm{~m} / \mathrm{s}-10 \mathrm{~km} / \mathrm{s})$. En effet, autour des années 1960, Shelton, Hendricks, Wuerker [18], Friichtenicht [19] puis d'autres ont développé des accélérateurs de microparticules dans le but de simuler l'impact de micrométéorites sur les vaisseaux et autres engins spatiaux, ainsi que dans le but de construire des détecteurs de micrométéorites embarqués à bord de satellites artificiels.

2. Dispositif expérimental. - Nous disposions d'un accélérateur électrostatique capable de communiquer des vitesses de quelques dizaines à quelques centaines de $\mathrm{m} / \mathrm{s}$ à des microparticules sphériques de fer carbonyle de quelques microns. Cet appareil, construit suivant le même principe que celui de Shelton et al. [18], a déjà été décrit en détail [20]. Les microparticules chargées positivement par contact sont accélérées entre la haute tension $U(0-150 \mathrm{kV})$ et

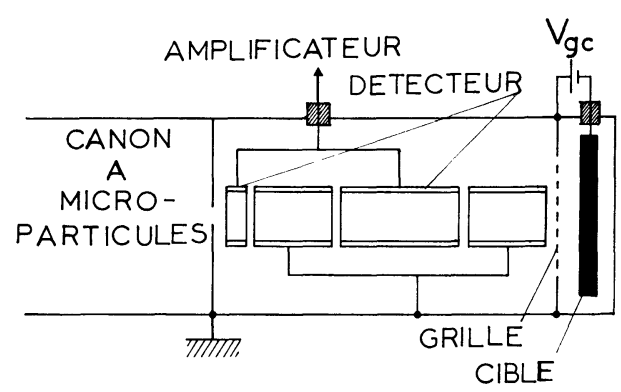

Fig. 1. - Dispositif expérimental utilisé pour l'étude de la réflexion des microparticules.

[Experimental arrangement for microparticle bouncing studies.]

la masse, avant de traverser, dans une zone à champ nul, un détecteur électrostatique constitué de deux cylindres successifs de longueur différente, chacun avec cylindres de garde (Fig. 1). Après avoir franchi une grille reliée à la masse et constituée de fils parallèles d'acier inoxydable de $0,1 \mathrm{~mm}$ de diamètre (transparence $T=0,9$ ), les microparticules viennent frapper l'électrode cible. Cette dernière était soumise à un champ électrique nul ou faible

$$
\left(-2 \times 10^{5}<E<+2 \times 10^{5} \mathrm{~V} / \mathrm{m}\right)
$$

appliqué entre la grille et la cible. La distance grillecible était typiquement de $5 \mathrm{~mm}$ pour une tension grille-cible $V_{\mathrm{gc}}$ comprise entre $\pm 1 \mathrm{kV}$. $V_{\mathrm{gc}}$ n'a donc aucun effet sensible sur la vitesse incidente $v_{1}$ ou sur la vitesse réfléchie $v_{2}$ de la microparticule. $\mathrm{Si} V_{\mathrm{gc}}$ n'était pas négligeable devant $U$, il serait aisé d'en tenir compte. L'ensemble était placé dans une enceinte à l'intérieur de laquelle régnait un vide industriel $\left(10^{-5}\right.$ torr $)$.

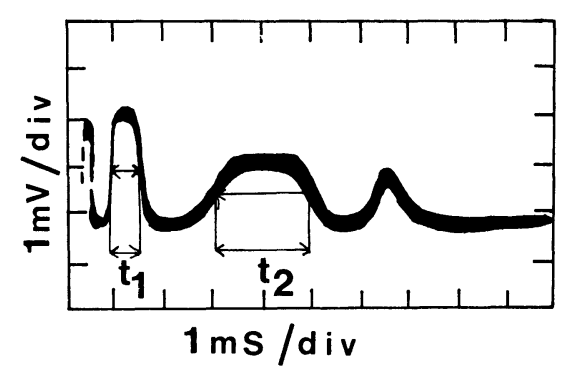

Fig. 2. - Signal électrique issu du détecteur montrant la réflexion d'une microparticule $\left(v_{2}<v_{1}\right)$. La dissymétrie du signal permet de distinguer le sens de propagation.

[Electrical signal from the detector showing microparticle bouncing $\left(v_{2}<v_{1}\right)$. Asymmetry of the signal enables the determination of the motion direction.]

Le signal dissymétrique issu du détecteur est amplifié et recueilli sur un oscilloscope cathodique à mémoire de grande sensibilité $(10 \mu \mathrm{V} /$ div $)$. La sensibilité du détecteur est d'environ $10^{-15} \mathrm{C}$. $\mathrm{Ce}$ signal (Fig. 2) permet de mesurer le temps de transit à travers le détecteur (largeur du signal à mi-hauteur) 
de la microparticule incidente $t_{1}$ ou réfléchie $t_{2}$, ainsi que les charges incidentes $q_{1}$ et réfléchies $q_{2}$. On en déduit :

$$
v_{1}=\frac{l}{t_{1}} \quad \text { et } \quad v_{2}=\frac{l}{t_{2}}
$$

\section{$l:$ longueur du détecteur}

ainsi que le coefficient de restitution $e$

$$
e=\frac{v_{2}}{v_{1}}=\frac{t_{1}}{t_{2}}
$$

Les relations

$$
\frac{1}{2} m v_{1}^{2}=q_{1} U
$$

et

$$
m=\frac{4}{3} \pi R^{3} \rho
$$

$m:$ masse de la microparticule,

$\rho$ : masse volumique de la microparticule,

permettent de déduire la masse et le rayon de la microparticule.

Nous avons étudié l'impact sur des cibles d'aluminium, de nickel, de cuivre et d'or. Ces électrodes étaient préparées par usinage au tour, puis par polissage à l'abrasif. La finition était obtenue par polissage à l'alumine de taille décroissante. Les électrodes étaient ensuite soigneusement lavées aux ultra-sons, dégraissées à l'alcool absolu et finalement séchées à l'étuve.

3. Résultats expérimentaux. - Nous avons, dans un premier temps, étudié la variation de la vitesse réfléchie en fonction de la vitesse incidente, les charges incidentes et réfléchies de la microparticule ne servant que comme moyen de détermination des vitesses incidentes et réfléchies. La figure 3 représente le pourcentage de microparticules qui rebondissent en fonction du champ électrique appliqué. Ce pourcentage est indépendant du champ. Nous avons observé en moyenne que $54 \%$ des microparticules rebondissaient sur une cible d'aluminium, soit $67 \%$, en tenant compte de la transparence de la grille. Il y a trois raisons pour lesquelles on peut ne pas détecter le retour d'une microparticule :

1) la microparticule peut rester collée sur la cible mais cet événement est peu fréquent compte tenu du domaine de vitesses incidentes; l'observation au microscope électronique à balayage (M. E. B.) ne montre d'ailleurs que quelques rares microparticules adhérant à la cible ;

2) la microparticule peut rebondir avec une charge inférieure au seuil de sensibilité du détecteur ;

3) enfin, il peut y avoir rebondissement dans une direction telle que la microparticule ne repasse pas à travers le détecteur.

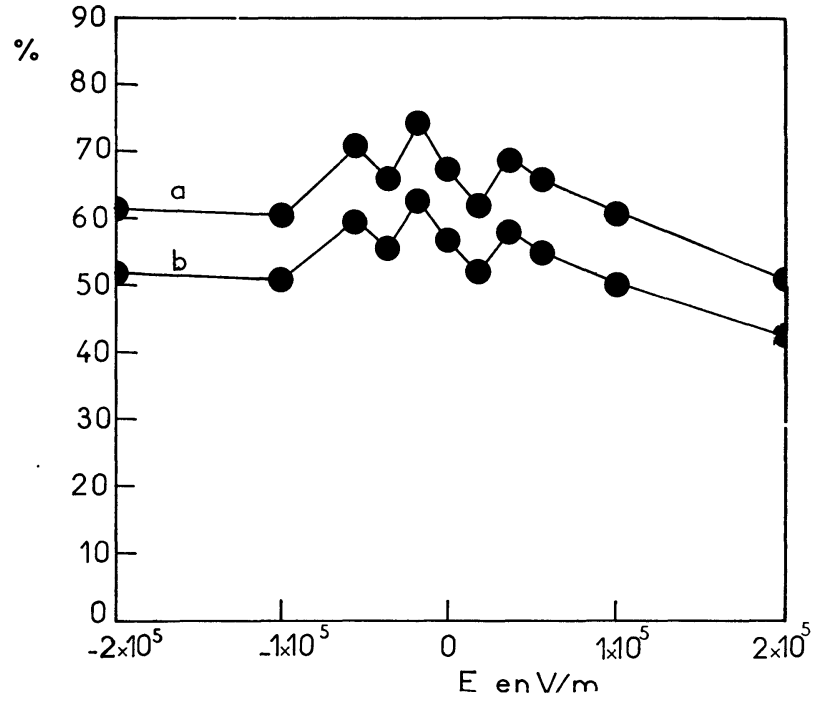

FIG. 3. - Pourcentage du nombre de microparticules qui rebondissent sur une cible d'aluminium en fonction du champ électrique appliqué. a : \% corrigé par la transparence de la grille; $b$ : \% mesuré.

[Percentage of microparticles bouncing on an aluminium target versus applied electrical field.]

Bien que l'incidence macroscopique, compte tenu $\mathrm{du}$ dispositif expérimental, soit quasiment normale (dispersion de la zone d'impact de $2 \mathrm{~mm}$ pour un parcours de $15 \mathrm{~cm}$ ), l'incidence réelle sur le microrelief des électrodes peut être différente. Un angle d'incidence supérieur à 4030 est suffisant pour que la microparticule réfléchie passe hors du détecteur. Toutefois, une étude d'impact en champ électrique élevé $\left(v_{\mathrm{gc}}=25 \mathrm{kV}\right)$ a montré un pourcentage beaucoup plus élevé de microparticules rebondissant. La seconde raison semble donc plus fréquente que la dernière. Par suite, les vitesses incidentes et réfléchies sont bien dirigées suivant l'axe du montage et du détecteur et ce dernier mesure les vitesses réelles des microparticules et non pas seulement leur composante axiale (l'erreur serait au maximum de 0,3\%).

On observe une assez grande dispersion des résultats, qui ne s'explique pas toujours par la précision des mesures. La détermination d'une vitesse se fait au mieux à $10 \%$ près. L'erreur sur le coefficient de restitution, rapport de deux longueurs relevées sur un même oscillogramme, est de l'ordre de $15 \%$.

La précision est souvent plus mauvaise encore. Nos mesures n'ont donc qu'une signification statistique et on ne peut parler que de comportement moyen. Sur les courbes, nous avons représenté la valeur moyenne d'une série de mesures, chaque barre d'erreur représentant l'écart-type.

La figure 4 montre, pour une même vitesse incidente de $66 \mathrm{~m} / \mathrm{s}$, la dispersion des vitesses réfléchies pour une cible d'aluminium. On observe d'abord une croissance rapide de la vitesse réfléchie en fonction de la vitesse incidente, puis une croissance moins rapide qui, si elle n'est pas linéaire, présente une 


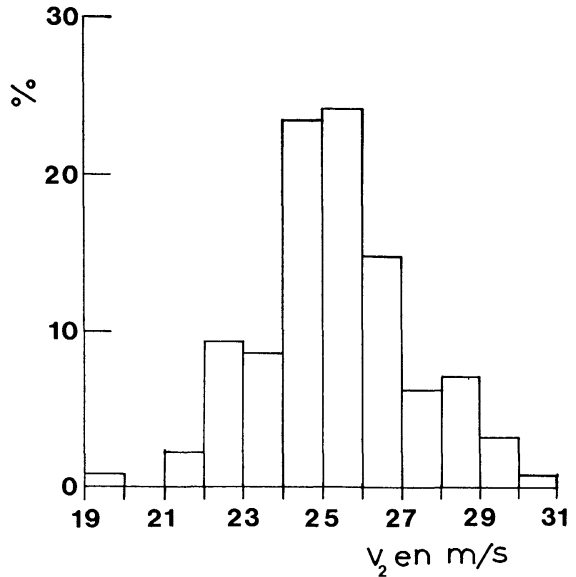

Fig. 4. - Dispersion des vitesses des microparticules réfléchies pour une même vitesse incidente de $66 \mathrm{~m} / \mathrm{s}$ pour une cible d'aluminium.

[Dispersion of the velocities of reflected microparticles for the same impact velocity $(66 \mathrm{~m} / \mathrm{s})$. Aluminium target.]

courbure peu accentuée. On en déduit que le coefficient de restitution décroît quand la vitesse incidente augmente. Les figures 5, 6, 7, 8 montrent ces variations pour l'aluminium et l'or. Le comportement des autres matériaux $\mathrm{Ni}, \mathrm{Cu}$, est identique et les courbes et les valeurs obtenues sont très voisines. On peut en déduire la perte relative d'énergie cinétique $1-e^{2}$ pendant le choc. Elle est sensiblement constante compte tenu des faibles variations de $e$ dans un large domaine de vitesses. Nous avons d'ailleurs trouvé directement ce résultat en établissant statistiquement une corrélation entre l'énergie de la microparticule réfléchie et les caractéristiques de la microparticule incidente : l'énergie des microparticules réfléchies est proportionnelle

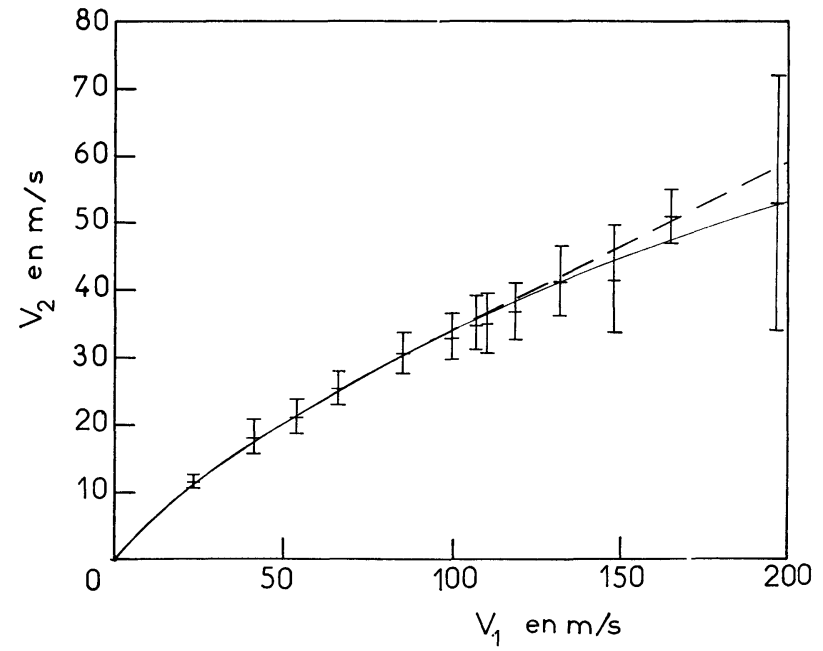

Fig. 5. - Variation de la vitesse réfléchie en fonction de la vitesse incidente pour une cible en aluminium. - - - approximation de Tabor ; - nos calculs.

[Variation of the velocity after reflection with the impact velocity. Aluminium target. .... Tabor's approximation. - our calculations.]

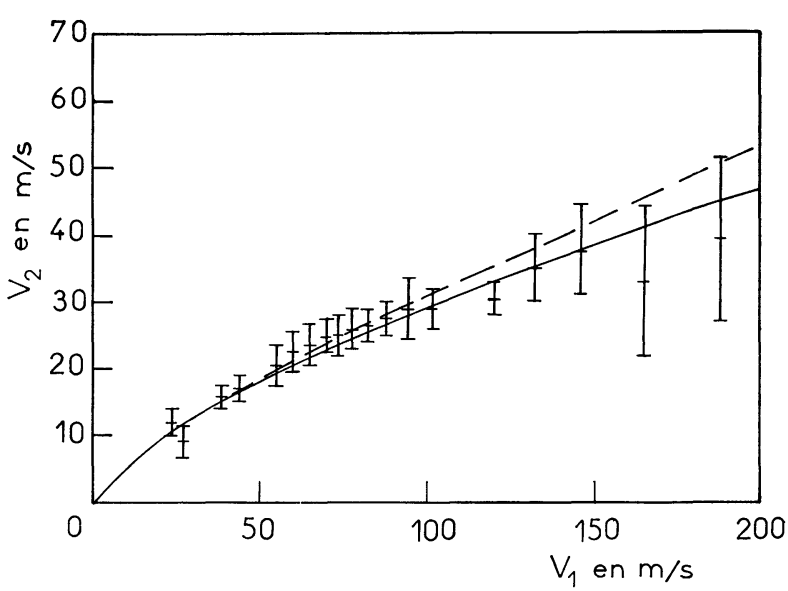

Fig. 6. - Variation de la vitesse réfléchie en fonction de la vitesse incidente pour une cible d'or. ...- approximation de Tabor; - nos calculs.

[Variation of the velocity after reflection with the impact velocity. Gold target. - . - - Tabor's approximation. —— our calculations.]

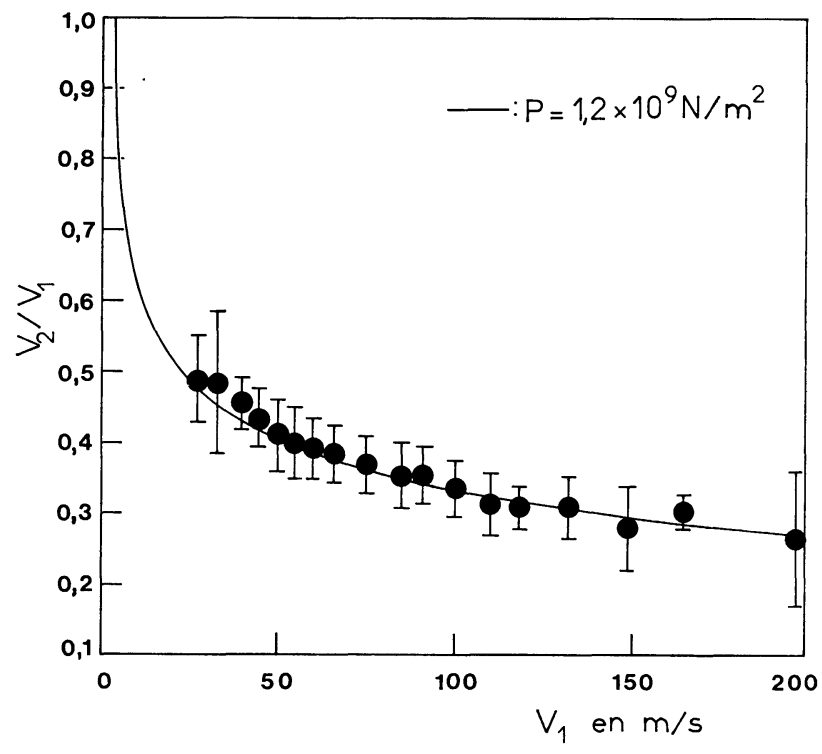

Fig. 7. - Variation du coefficient de restitution en fonction de la vitesse incidente pour une cible en aluminium.

[Variation of the coefficient of restitution with the impact velocity. Aluminium target.]

à l'énergie des microparticules incidentes (Fig. 9). Ce résultat n'est pas contradictoire avec la décroissance de $e$ en fonction de la vitesse incidente car $e^{2}$ est petit devant 1 et les variations de $e$ influent peu sur la quantité $1-e^{2}$. A une vitesse incidente de $100 \mathrm{~m} / \mathrm{s}$ correspond une perte d'énergie de $90 \%$ pratiquement indépendante de la nature de la cible.

L'observation au M. E. B. des différentes cibles montre la présence de très nombreuses empreintes sensiblement sphériques. Ceci prouve que le seuil de déformation plastique est dépassé. La figure 10 représente une empreinte de $2 \mu \mathrm{m}$ de diamètre sur une cible d'or. La figure 11 montre une empreinte 


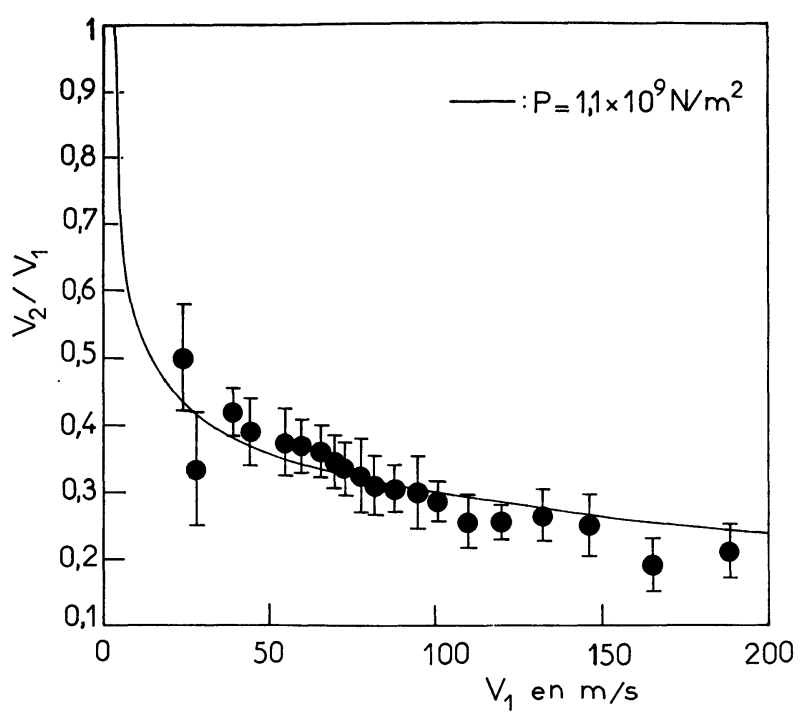

Fig. 8. - Variation du coefficient de restitution en fonction de la vitesse incidente pour une cible d'or.

[Variation of the coefficient of restitution with the impact velocity. Gold target.]

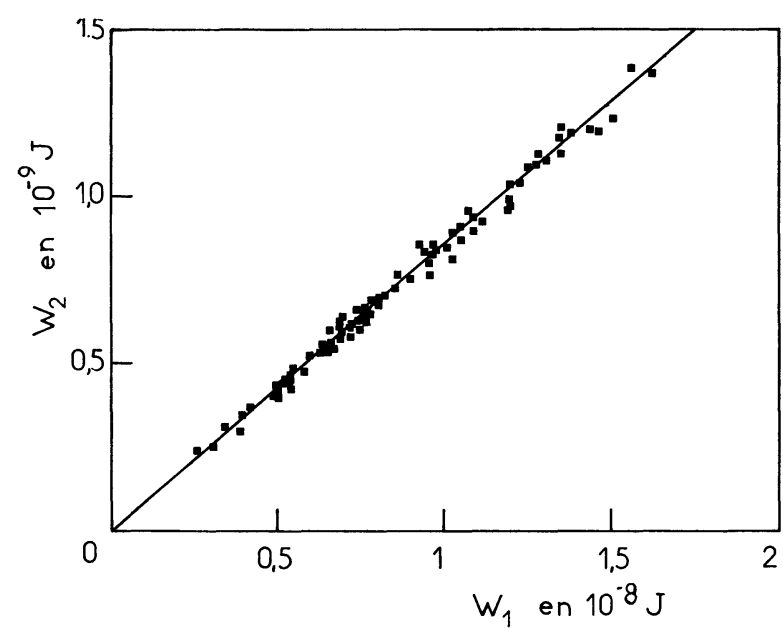

Fig. 9. - Energie de la microparticule réfléchie en fonction de l'énergie de la microparticule incidente (cible en cuivre).

[Kinetic energy of a reflected microparticle versus kinetic energy before impact (copper target).]

de $3,8 \mu \mathrm{m}$ de diamètre sur une cible d'aluminium. La déformation des raies de polissage est très nette dans les deux cas. La figure 12 représente un carré de $33 \mu \mathrm{m}$ de côté sur une cible de cuivre. La densité des empreintes y est importante $\left(\simeq 3 \times 10^{10}\right.$ par $\left.\mathrm{m}^{2}\right)$. Avec le matériel dont nous disposions, il n'était pas possible d'établir une relation directe entre une microparticule incidente donnée et l'empreinte correspondante. On ne peut qu'établir une relation globale entre l'ensemble des empreintes observées et la distribution des vitesses incidentes. Dans ces conditions, nous n'avons pas pu déterminer avec précision la vitesse critique $v_{\mathrm{c}}$ à partir de laquelle il y a déformation plastique de la cible.

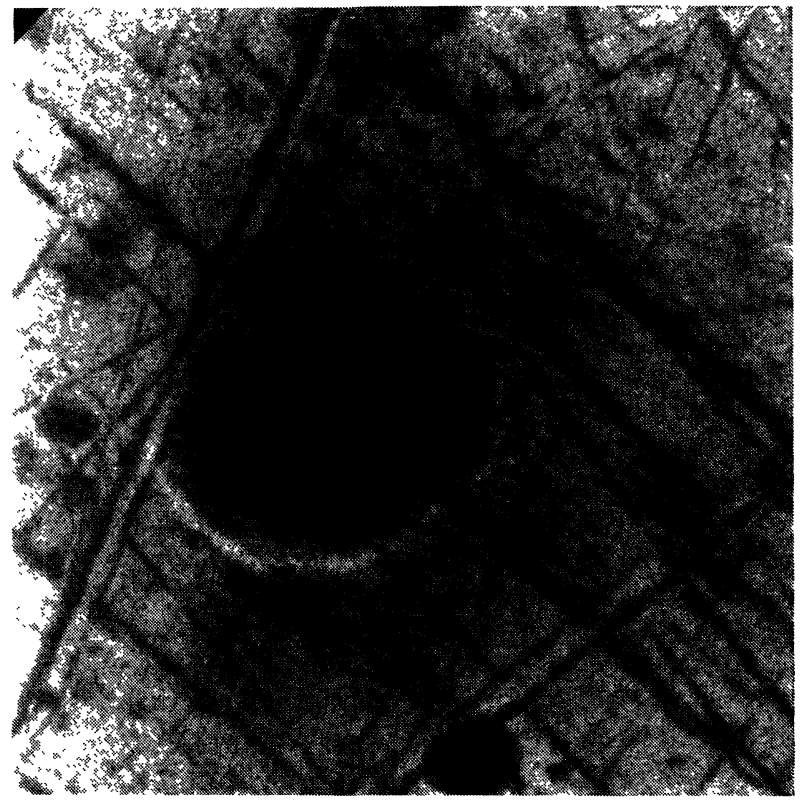

FIG. 10. - Empreinte de $1,85 \mu \mathrm{m} \times 2,22 \mu \mathrm{m}$ observée au M. E. B. sur une électrode en or.

$\left[1.85 \times 2.22 \mu \mathrm{m}^{2}\right.$ indentation on a gold target observed with a scanning electron microscope (S. E. M.).]

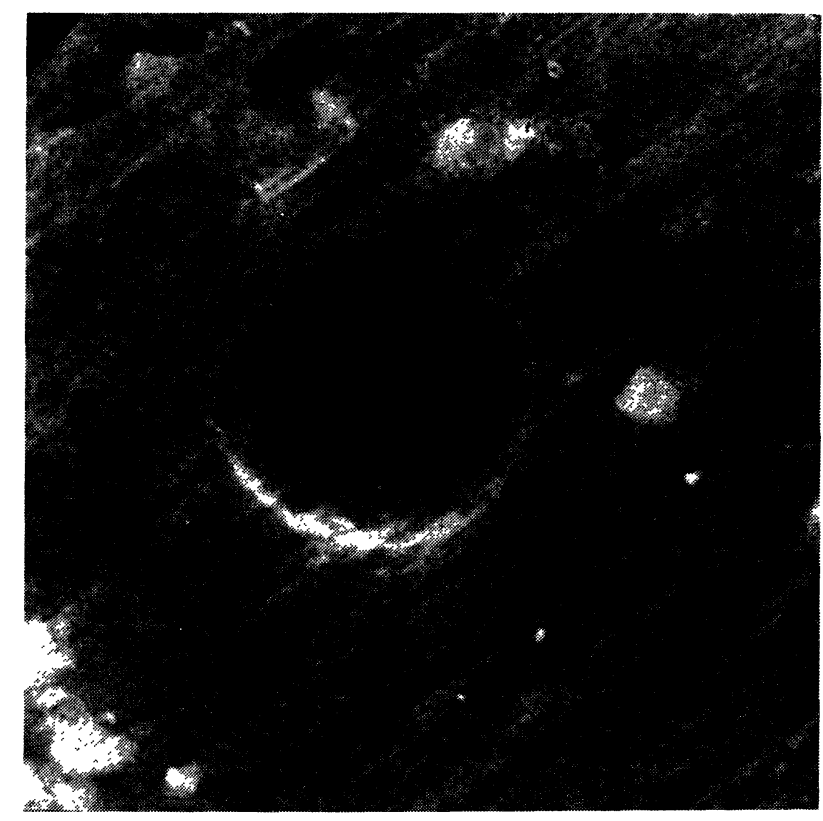

FIG. 11. - Empreinte sphérique de 3,8 $\mu \mathrm{m}$ de diamètre observée au M. E. B. sur une électrode en aluminium.

[Spherical indentation $3.8 \mu \mathrm{m}$ in diameter on an aluminium target (S. E. M. observation).]

4. Discussion et interprétation des résultats. - $\mathrm{Si}$ la vitesse incidente de la microparticule est inférieure à $v_{\mathrm{c}}$, le choc est élastique. La théorie en a été faite pour la première fois par Hertz [21]. $v_{c}$ est faible pour des billes macroscopiques $(0,1 \mathrm{~m} / \mathrm{s})$ [22]; elle est plus élevée dans le cas des microparticules. $v_{c}$ est liée à la limite d'élasticité $P$ par la relation [23] :

$$
v_{\mathrm{c}}=K P^{5 / 2} \text {. }
$$




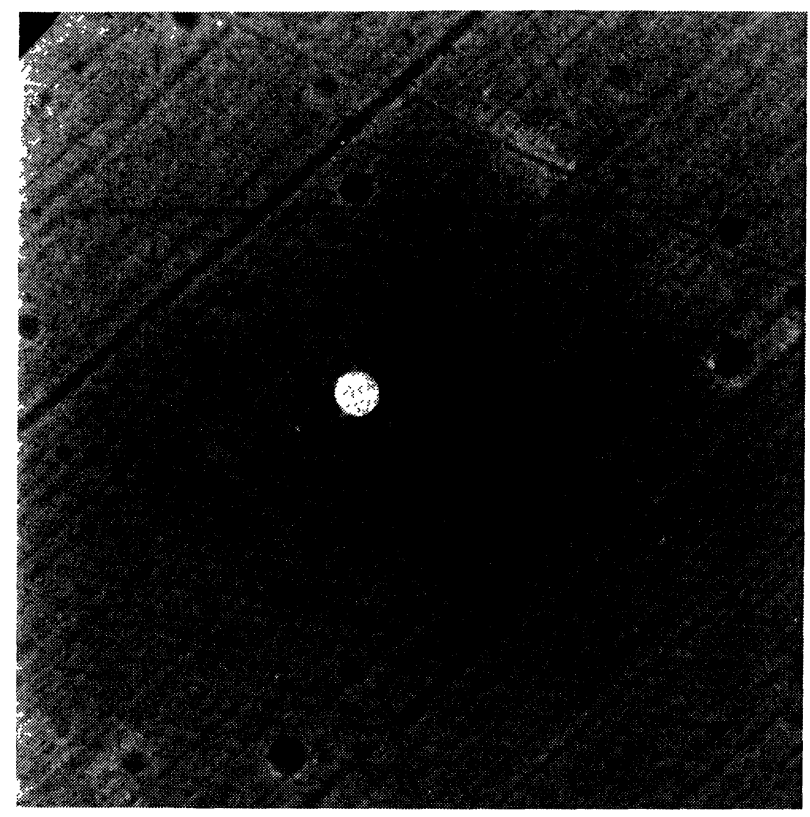

FIG. 12. - Distribution des empreintes formées par les microparticules sur une cible de cuivre (côté du carré : $33 \mu \mathrm{m}$ ).

[Distribution of indentations produced by microparticle impact on a copper target. Imaged area : $33 \times 33 \mu \mathrm{m}^{2}$.]

Nous verrons par la suite que la valeur de $P$ pour l'impact de microparticules est multipliée par un facteur de 4 à 5 par rapport à la limite d'élasticité obtenue par compression ou étirement. Ceci conduit à une valeur de $v_{\mathrm{c}}$ inférieure à $10 \mathrm{~m} / \mathrm{s}$, ce qui est en accord avec nos observations au M. E. B. $v_{c}$ étant liée aux caractéristiques mécaniques de la cible dépend $\mathrm{du}$ traitement des électrodes (usinage, polissage...) et de l'état mécanique du point d'impact effectif, d'où probablement une certaine dispersion de sa valeur.

Pour les vitesses supérieures à $v_{c}$ l'étude du rebondissement des microparticules est identique à l'étude de la dureté par la méthode du scléroscope. On distingue trois étapes dans le choc: une étape de déformation élastique tant que la pression est inférieure à $P$ (pression dynamique à partir de laquelle la déformation plastique commence), puis une étape de déformation plastique jusqu'à ce que la vitesse de la microparticule s'annule, et enfin la séparation de la microparticule de la cible sous l'action des forces de déformation élastique.

\section{Soient :}

$R_{1} \quad$ : rayon de la microparticule.

$\boldsymbol{R}_{\mathbf{2}} \quad$ : rayon de courbure de l'empreinte supposée sphérique sur la cible.

$M \quad$ : masse de la microparticule.

$\rho \quad:$ masse volumique de la microparticule.

$a$ et $h$ : rayon et profondeur de l'empreinte sur la cible (Fig. 13).

$E_{1}, E_{2}$ : modules d'Young de la microparticule et de la cible.

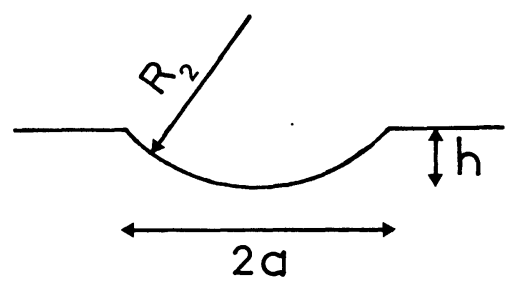

FIG. 13. - Schéma d'une empreinte.

[Schematic representation of an indentation.]

$v_{1}, v_{2}:$ les vitesses de la microparticule incidente et réfléchie.

$W_{1}, W_{2}$ : les énergies cinétiques de la microparticule incidente et réfléchie.

Le volume $V$ de la déformation permanente s'écrit

$$
V=\pi h^{2}\left(R_{2}-\frac{h}{3}\right)
$$

avec

$$
h=R_{2}\left[1-\left(1-\frac{a^{2}}{R_{2}^{2}}\right)^{1 / 2}\right] .
$$

Faisons l'hypothèse que $P$ reste constant pendant la déformation (Martel [24], Andrews [22], Tabor [23]). On peut donc écrire que :

$$
W_{1}-W_{2}=P \times V \text {. }
$$

De la théorie de l'élasticité de Hertz, on tire la relation

$$
\frac{1}{R_{2}}=\frac{1}{R_{1}}-0,69 \frac{P \pi}{a}\left(\frac{1}{E_{1}}+\frac{1}{E_{2}}\right) .
$$

D'après Prescott [25] et Tabor [23]

$$
v_{2}^{2}=\frac{0,54 \times \pi^{2} P^{2} a^{3}}{M}\left(\frac{1}{E_{1}}+\frac{1}{E_{2}}\right) .
$$

Dans ces expressions, le coefficient de Poisson a été considéré comme constant et égal à 0,3 .

Tabor [23] qui étudiait l'impact de billes animées de vitesses faibles (de l'ordre du $\mathrm{m} / \mathrm{s}$ ), a utilisé une expression simplifiée pour le volume de la déformation permanente

$$
V=\pi a^{4} / 4 R_{2} \text {. }
$$

Avec cette hypothèse simplificatrice, il démontre que :

$$
P^{5}=\frac{v_{2}^{8}}{\left(v_{1}^{2}-\frac{3}{8} v_{2}^{2}\right)^{3}} \cdot \frac{M}{218 R_{1}^{3}} \cdot \frac{1}{\left(\frac{1}{E_{1}}+\frac{1}{E_{2}}\right)^{4}}
$$

que l'on peut mettre sous la forme

$$
v_{1}^{2}=K^{8}\left[\frac{1}{e^{8}}-\frac{9}{8} \frac{1}{e^{6}}+\frac{27}{64} \frac{1}{e^{4}}-\frac{27}{512} \frac{1}{e^{2}}\right]
$$

avec

$$
K=\left[163,5 \frac{P^{5}}{\pi \rho}\left(\frac{1}{E_{1}}+\frac{1}{E_{2}}\right)^{4}\right]^{1 / 8}
$$


Nous avons calculé numériquement cette relation. Pour les vitesses inférieures à $100 \mathrm{~m} / \mathrm{s}$, les courbes obtenues coïncident bien avec les résultats expérimentaux (Figs. 5 et 6 ). Pour les vitesses plus élevées, les valeurs expérimentales des vitesses réfléchies sont inférieures à celles prévues par la relation de Tabor. On déduit de ces courbes une valeur expérimentale de $P$. Elle est de $1,2 \times 10^{9} \mathrm{~N} \times \mathrm{m}^{-2}$ pour l'aluminium. La valeur de $P_{\mathrm{m}}$ (pression statique qui donnerait la même déformation permanente) est liée à la limite d'élasticité $Y$ obtenue par tension ou compression par la relation (23) :

$$
P_{\mathrm{m}}=C Y, 2,6<C<3 .
$$

Tabor a montré expérimentalement que $P$ est supérieur d'environ $10 \%$ à $P_{\mathrm{m}} . Y=10^{8} \mathrm{~N} / \mathrm{m}^{2}$ pour l'aluminium. La valeur microscopique expérimentale de $P$ est donc environ 4 fois plus élevée que la valeur macroscopique. Ce comportement se retrouve avec les autres métaux étudiés. Il peut s'expliquer par le fait que les méthodes classiques mesurent une valeur moyenne pour une surface polycristalline comportant plus ou moins de défauts alors que dans nos expériences, vu la taille des microparticules, l'impact se fait pratiquement sur un monocristal. Or les monocristaux possèdent des qualités mécaniques bien supérieures. Il est probable que la dispersion des résultats est liée à une certaine dispersion dans les valeurs locales de $P$ liée à toute l'histoire des électrodes. L'écrouissage de la couche superficielle du métal peut également expliquer cette limite d'élasticité anormalement élevée.

L'écart constaté pour les vitesses les plus élevées entre les valeurs déduites des expressions de Tabor et les valeurs expérimentales est dû à l'approximation sur le volume qui demeure valable tant que ce dernier est petit, c'est-à-dire tant que la vitesse reste faible. Sans cette hypothèse simplificatrice, on dispose des relations (2), (3), (4), (5) et (6). Comme il n'est pas possible de trouver de relations simples dans le cas général, nous avons résolu numériquement ces équations : pour une microparticule on se donne $a$ et on en déduit successivement $R_{2}, h, V, v_{2}, v_{1}$ et $e$. Cette méthode permet d'accéder, pour une valeur donnée de la vitesse incidente, à la vitesse réfléchie, au coefficient de restitution ainsi qu'aux caractéristiques de l'empreinte : rayon de courbure, diamètre et profondeur. Sur les figures 6 et 7 on trouve bien que nos courbes sont confondues avec celles de Tabor pour les faibles vitesses et qu'elles s'en écartent sensiblement pour les vitesses plus élevées pour lesquelles elles sont en meilleur accord avec l'expérience. Ces équations vérifient bien que le coefficient de restitution est indépendant du rayon de la microparticule et qu'il varie en sens inverse de la vitesse incidente. La figure 14 représente la variation du rapport du rayon de courbure de l'empreinte au rayon de la microparticule en fonction de la vitesse

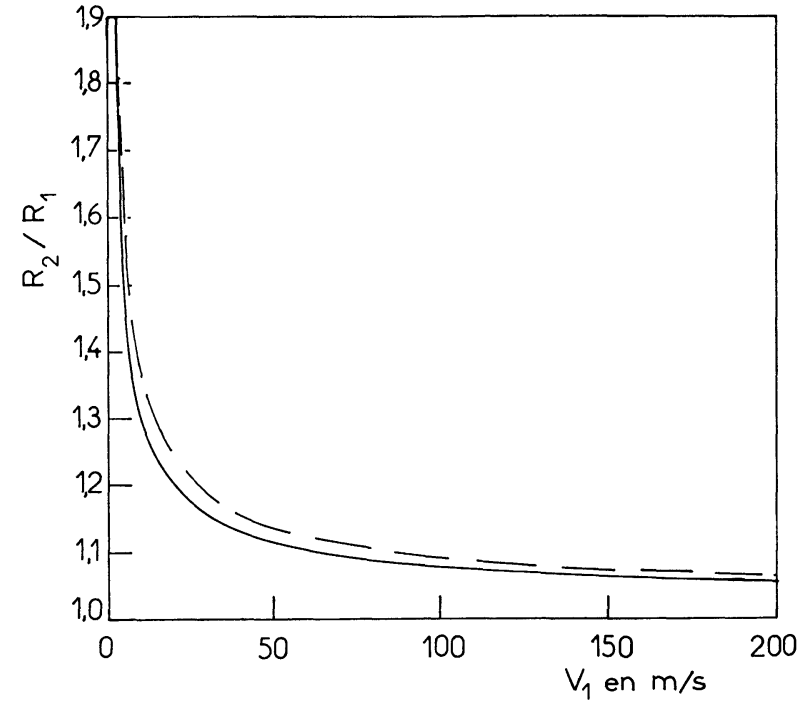

Fig. 14. - Rapport du rayon de courbure de l'empreinte sur de l'aluminium au rayon de la microparticule de fer en fonction de la vitesse incidente $\left(P=1,1 \times 10^{9} \mathrm{~N} / \mathrm{m}^{2}\right)$.

[Ratio of the curvature radius of an indentation to the microparticle radius versus impact velocity $\left(P=1.1 \times 10^{9} \mathrm{~N} / \mathrm{m}^{2}\right)$.]

incidente. Ce rapport décroît rapidement pour les vitesses inférieures à $50 \mathrm{~m} / \mathrm{s}$, vitesse pour laquelle il est de 1,1, puis tend lentement vers 1 .

$\mathrm{Vu}$ l'accord assez bon, tant qualitatif que quantitatif, entre les valeurs expérimentales et théoriques, les relations intermédiaires donnant les caractéristiques de la déformation permanente peuvent être considérées comme correctes. Nous avons tracé les courbes donnant le diamètre $D$ et la profondeur $h$ de l'empreinte pour une cible en aluminium. Sur ces graphes nous avons également reporté les valeurs expérimentales de Rudolph [17]. Les valeurs de $P$ étant sensiblement identiques pour les différentes cibles, nous nous limiterons aux valeurs numériques pour l'aluminium qui permettent une comparaison avec Rudolph.

4.1 Diamètre De L'EMPReinTE. - Bien que les relations ne soient pas rigoureusement linéaires, on peut, avec une bonne approximation, comme le montrent les figures 15 et 16 , mettre le diamètre $D$ de l'empreinte sous la forme

$$
D=C_{1} R_{1} v_{1}^{\alpha_{1}}
$$

$\alpha_{1}$ est voisin de 0,48 . On peut en première approximation le prendre égal à 0,5 . Il est indépendant du matériau considéré (donc de $P$ ) et décroît légèrement pour les vitesses supérieures à $200 \mathrm{~m} / \mathrm{s}$.

$C_{1} \simeq 13 \times 10^{-2}$ U. S. I. $C_{1}$ est fonction du matériau. Il est lié à la limite d'élasticité par la relation

$$
C_{1}=K_{1} P^{-0,25} \text {. }
$$

La limite de validité de nos calculs est atteinte quand le diamètre de contact est égal au diamètre 


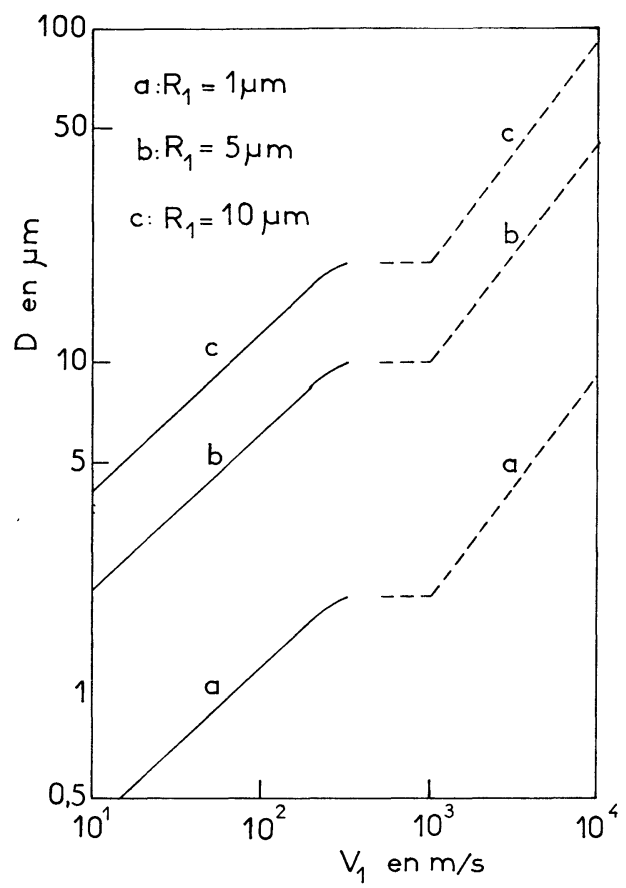

FIG. 15. - — Valeur calculée du diamètre de l'empreinte sur de l'aluminium en fonction de la vitesse incidente $(0-310 \mathrm{~m} / \mathrm{s})$ pour des microparticules de 1,5 et $10 \mu \mathrm{m}$ de rayon $\left(P=1,1 \times 10^{9} \mathrm{~N} / \mathrm{m}^{2}\right) ;-$ - Résultats de Rudolph [17].

[- Computed variation of the indentation diameter on aluminium with the impact velocity $(0-310 \mathrm{~m} / \mathrm{s})$. Microparticle radius : 1 to $5 \mu \mathrm{m}\left(P=1.1 \times 10^{9} \mathrm{~N} / \mathrm{m}^{2}\right)$. . - Rudolph's results [17].]

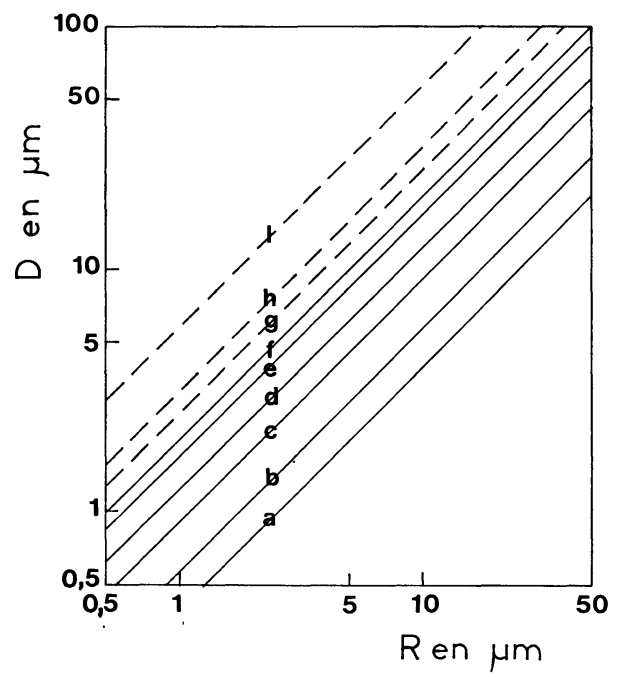

Fig. 16. - — Valeur calculée du diamètre de l'empreinte sur de l'aluminium en fonction du rayon de la microparticule pour diverses vitesses incidentes $(0-310 \mathrm{~m} / \mathrm{s}) ; \mathrm{a}: v_{1}=10 \mathrm{~m} / \mathrm{s}$; $\mathrm{b}: v_{1}=20 \mathrm{~m} / \mathrm{s} ; \quad$ c : $v_{1}=50 \mathrm{~m} / \mathrm{s} ; \quad \mathrm{d}: v_{1}=100 \mathrm{~m} / \mathrm{s}$; e : $v_{1}=200 \mathrm{~m} / \mathrm{s} ;$ f : $v=310 \mathrm{~m} / \mathrm{s}$. ... Résultats de Rudolph [17]. (g: $v_{1}=1,5 \mathrm{~km} / \mathrm{s} ; \mathrm{h}: v_{1}=2 \mathrm{~km} / \mathrm{s}$; i : $\left.v_{1}=5 \mathrm{~km} / \mathrm{s}\right)$.

[- Computed variation of the indentation diameter on aluminium with the microparticle radius for various impact velocities $(0-310 \mathrm{~m} / \mathrm{s}) . .$. . Rudolph's results [17].] de la microparticule $\left(a=R_{1}\right)$. La force de contact est alors maximum et vaut $P \pi R_{1}^{2}$. La vitesse incidente correspondante est de $310 \mathrm{~m} / \mathrm{s}$. On peut admettre que pour les vitesses supérieures, les 2 diamètres sont égaux.

Rudolph [17] a montré expérimentalement que le diamètre du cratère était égal au diamètre de la microparticule pour des vitesses comprises entre 500 et $1000 \mathrm{~m} / \mathrm{s}$. Pour les vitesses supérieures :

$$
D=C_{1}^{\prime} R_{1} v_{1}^{\alpha_{1}^{\prime}}, \quad \alpha_{1}^{\prime} \simeq \frac{2}{3}, \quad C_{1}^{\prime}=1,98 \times 10^{-2} .
$$

On a donc le même type de relation avec des constantes différentes. On constate également un bon raccordement entre les deux domaines de vitesse. Enfin, ces valeurs sont en accord avec la taille des empreintes observées au M. E. B.

Nous avons représenté figure 19 le diamètre de l'empreinte calculé à partir de l'hypothèse simplificatrice de Tabor. On est conduit à un diamètre plus grand et surtout celui-ci devient égal au diamètre de la microparticule pour une vitesse incidente de $230 \mathrm{~m} / \mathrm{s}$ au lieu de $310 \mathrm{~m} / \mathrm{s}$.

4.2 Profondeur DU CRATÈRE. - Les figures 17 et 18 montrent que la profondeur $h$ du cratère est proportionnelle au rayon de la microparticule et à la vitesse incidente à la puissance $\alpha_{2}$

$$
h=C_{2} R_{1} v_{1}^{\alpha_{2}}
$$

$\alpha_{2}$ est voisin de 1,1 . Comme $\alpha_{1}$, il est indépendant de la nature de la cible ; par contre, il croît légèrement pour les vitesses supérieures à $200 \mathrm{~m} / \mathrm{s}$.

$C_{2}=1,3 \times 10^{-3}$ U. S. I. $C_{2}$ dépend de la nature

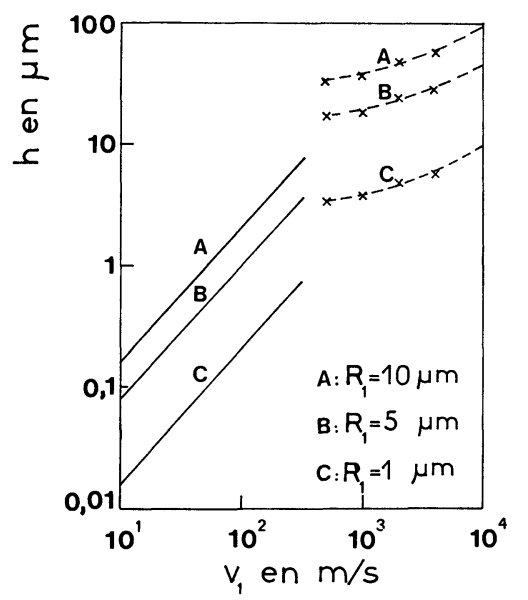

FIG. 17. - - Valeur calculée de la profondeur de l'empreinte laissée par une microparticule de 1,5 et $10 \mu \mathrm{m}$ de rayon sur une cible en $\mathrm{Al}$ en fonction de la vitesse incidente $(0-310 \mathrm{~m} / \mathrm{s})$; -..- Résultats de Rudolph [17].

[- Computed variation of the indentation depth on an aluminium target with the impact velocity $(0-310 \mathrm{~m} / \mathrm{s})$. Microparticle radius : 1-5 $\mu \mathrm{m}$. ...- Rudolph's results [17].] 


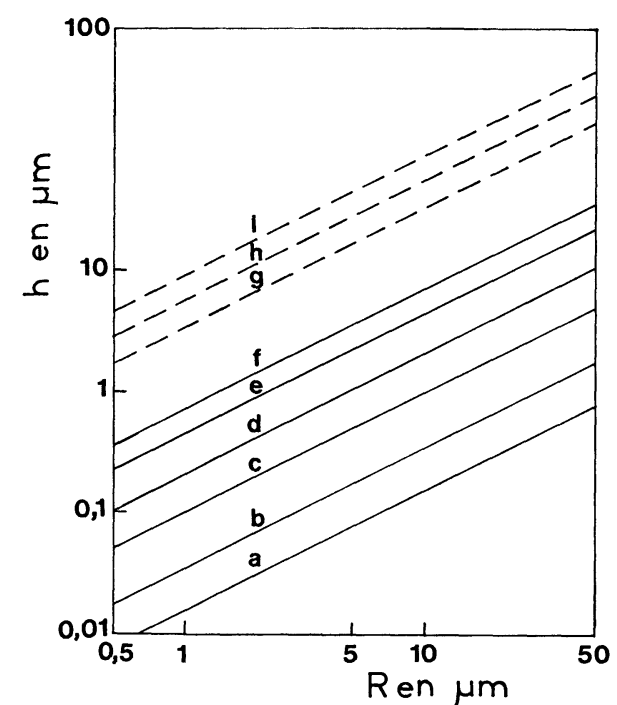

FIG. 18. - — Valeur calculée de la profondeur de l'empreinte laissée sur une cible d'Al en fonction du rayon de la microparticule pour diverses valeurs de la vitesse incidente; a : $v_{1}=10 \mathrm{~m} / \mathrm{s} ; \quad$ b : $v_{1}=20 \mathrm{~m} / \mathrm{s} ; \quad$ c : $v_{1}=50 \mathrm{~m} / \mathrm{s}$; $\mathrm{d}: v_{1}=100 \mathrm{~m} / \mathrm{s} ;$ e : $v_{1}=200 \mathrm{~m} / \mathrm{s} ; \mathrm{f}: v_{1}=300 \mathrm{~m} / \mathrm{s}$; ... - Résultats de Rudolph [17]. g: $v_{1}=500 \mathrm{~m} / \mathrm{s}$;

$$
\mathrm{h}: v_{1}=4 \mathrm{~km} / \mathrm{s} ; \mathrm{i}: v_{1}=10 \mathrm{~km} / \mathrm{s} \text {. }
$$

[- Computed variation of the indentation depth on an aluminium target with the microparticle radius for various impact velocities $(0-310 \mathrm{~m} / \mathrm{s}) . . .-$ Rudolph's results [17].]

de la cible. $C_{2}$ est lié à la limite d'élasticité par la relation

$$
C_{2}=K_{2} P^{-2 / 3} .
$$

Rudolph [17] a montré que le rapport profondeur sur diamètre du cratère passe par un maximum et tend vers une constante égale à 1 pour l'aluminium. On observe donc dans la zone de transition une petite discontinuité.

La figure 19 montre que l'hypothèse simplificatrice de Tabor conduit à une valeur trop faible de la profondeur de l'empreinte.

\subsection{Volume Du CRatère. - Par hypothèse}

$$
v=\frac{W_{1}-W_{2}}{P}=\frac{W_{1}}{P}\left(1-e^{2}\right) .
$$

Pour les vitesses supérieures à quelques dizaines de $\mathrm{m} / \mathrm{s}$, le coefficient de restitution varie peu et $\left(1-e^{2}\right) / P$ est sensiblement constant (Tableau I). Donc

$$
V=C_{3} W_{1}, \quad C_{3}=\frac{1-e^{2}}{P} .
$$

Le volume de l'empreinte est proportionnel à l'énergie de la microparticule incidente. Ceci est en plein accord avec les résultats de Martel [24] et avec nos résultats sur la perte relative d'énergie, laquelle est constante. Le tableau I donne les variations de $C_{3}$ avec la vitesse incidente.

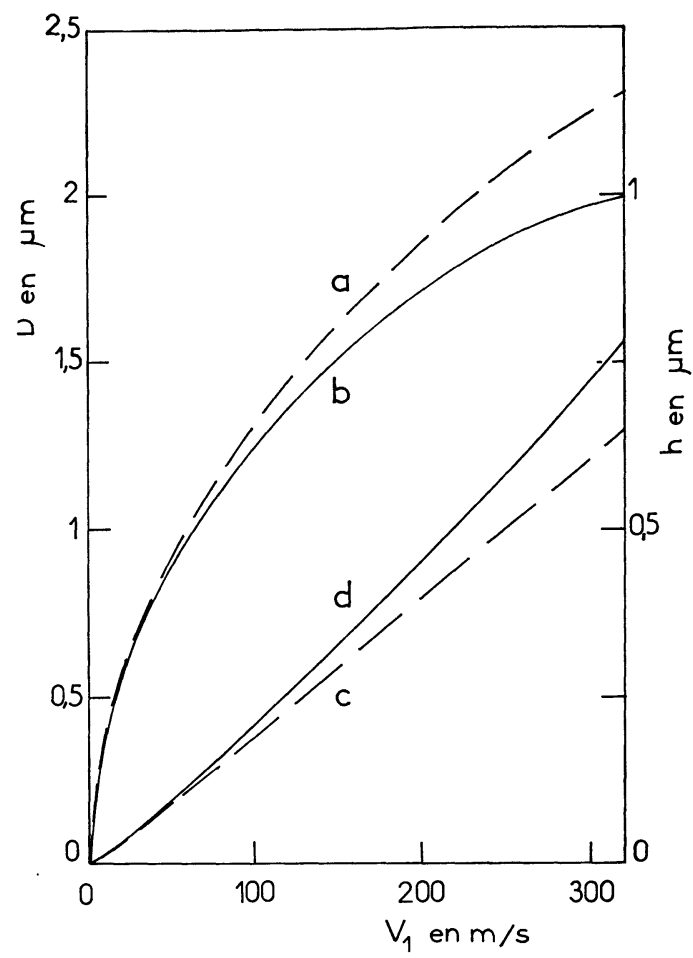

Fig. 19. - Diamètre $(a, b)$ et profondeur $(c, d)$ de l'empreinte laissée sur une cible d'Al par une microparticule de fer de $1 \mu \mathrm{m}$ de rayon en fonction de la vitesse incidente. $P=1,1 \times 10^{9} \mathrm{~N} / \mathrm{m}^{2}$. . - - approximation de Tabor ; - nos calculs.

[(a, b) Diameter and (c, d) depth of indentation produced by an iron microparticle (radius : $1 \mu \mathrm{m}$ ) versus impact velocity. $P=1.1 \times 10^{9} \mathrm{~N} / \mathrm{m}^{2}$. . . . Tabor's approximation; - our calculations.]

\section{TABLEAU I}

Valeurs du coefficient $C_{3}$ calculées à partir des valeurs théoriques $d u$ coefficient de restitution pour l'aluminium en fonction de la vitesse incidente des microparticules.

[Computed value of coefficient $C_{3}$ from theoretical values of the coefficient of restitution for aluminium as a function of the impact velocity of microparticles.]

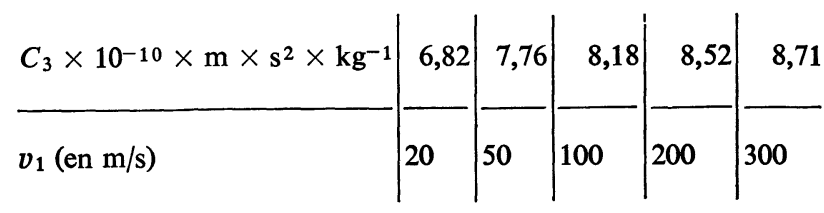

Ces résultats sont identiques à ceux de Rudolph [17]

$$
V=C_{3}^{\prime} W_{1}, C_{3}^{\prime}=3,4 \times 10^{-10} .
$$

Les valeurs de $C_{3}$ et $C_{3}^{\prime}$ sont du même ordre de grandeur. Cette relation est donc valable quel que soit le domaine de vitesse considéré.

Il faut toutefois remarquer que dans nos calculs nous avons négligé la déformation de la microparticule 
supposée implicitement indéformable. L'excellent accord avec les courbes expérimentales donnant la vitesse réfléchie en fonction de la vitesse incidente permet de penser que cette hypothèse est assez bien vérifiée, du moins dans le domaine $20-200 \mathrm{~m} / \mathrm{s}$. Pour des vitesses plus élevées, on atteindra rapidement la vitesse limite $v_{\mathrm{p}}$ au-dessus de laquelle il y a vaporisation partielle de la microparticule. $v_{\mathrm{p}}$ est donnée par la relation [26]

$$
v_{\mathrm{p}}=(8 P / \rho)^{1 / 2} .
$$

Enfin, Bowden et Tabor [27] ont montré que le temps de contact microparticule-cible est donné par la relation

$$
t=\frac{\pi}{2}\left(\frac{2}{3} \frac{\rho}{P}\right)^{1 / 2} R_{1} .
$$

Il intervient dans les échanges de charges électriques entre la microparticule et la cible.

5. Conclusions. - L'étude expérimentale de l'impact de microparticules de fer de la taille de quelques microns sur des cibles d'aluminium, de cuivre, de nickel, et d'or a montré une certaine dispersion des résultats mais le comportement moyen est identique à celui du choc de billes macroscopiques sur une cible. Nous avons mis en évidence que :

1. Le coefficient de restitution décroît quand la vitesse incidente croît. Il varie peu d'un métal à l'autre et il est indépendant du rayon des microparticules.
2. Ces résultats sont en accord avec les conclusions théoriques de Tabor [23] dans le cas du choc d'une bille macroscopique sur un plan. Celles-ci restent applicables au cas des microparticules à condition d'utiliser des formules rigoureuses pour le volume et la profondeur de la calotte sphérique constituant l'empreinte. Une résolution numérique devient alors nécessaire. On est toutefois conduit à une limite d'élasticité microscopique supérieure d'un coefficient de l'ordre 4 à celle mesurée macroscopiquement.

3. On peut, à partir des hypothèses et calculs précédents, en déduire les caractéristiques de l'empreinte laissée sur la cible :

- le diamètre est donné par la relation :

$$
D=K_{1} P^{-0,25} R_{1} v_{1}^{\alpha_{1}} \quad\left(\alpha_{1} \simeq 0,5\right)
$$

- la profondeur par :

$$
h=K_{2} P^{-2 / 3} R_{1} v_{1}^{\alpha_{2}} \quad\left(\alpha_{2}=1,1\right)
$$

- le volume de l'empreinte est proportionnel à l'énergie de la microparticule incidente :

$$
V=C_{3} W_{1}, \quad C_{3} \simeq 8 \times 10^{-10} .
$$

4. Ces résultats concordent avec ceux obtenus par Rudolph dans le domaine $500 \mathrm{~m} / \mathrm{s}-10 \mathrm{~km} / \mathrm{s}$.

5. La perte d'énergie est importante pour la microparticule $(90 \%)$. Le choc est donc fortement inélastique. Ceci est peu favorable à un gain d'énergie des microparticules par rebondissements successifs entre les électrodes, comme l'ont proposé Latham et Braun [14].

\section{Bibliographie}

[1] Maitland, A., J. Appl. Phys. 32 (1961) 2399-407.

[2] Alpert, D., LeE, D. A., Lyman, E. M., Tomaschke, H. E., J. Vac. Sci. Technol. 1 (1964) 35.

[3] Brodie, I., J. Appl. Phys. 35 (1964) 2324-32.

[4] Boyle, W. S., Kisliuk, P., Germer, L. H., J. Appl. Phys. 26 (1955) 720-25.

[5] Trump, J. G., van de Graff, R. J., J. Appl. Phys. 18 (1947) 327-32.

[6] Cranberg, L., J. Appl. Phys. 23 (1952) 518-22.

[7] Menon, M. M. and Srivastava, K. D., J. Appl. Phys. 45 (1974) 2094.

[8] Theophilus, G. D., Srivastava, K. D., van HeeswiJk, R. G., J. Appl. Phys. 47 (1976) 897-8.

[9] Texier, C., J. Phys. D : Appl. Phys. 10 (1977) 1693-1702.

[10] SuIvkov, I. N., Sov. Phys. Techn. Phys. 2 (1957) 1928-34.

[11] Rohrbach, F., Rapport du C. E. R. N., Genève 1971.

[12] Chatterton, P. A., Menon, M. M., Srivastava, K. D., J. Appl. Phys. 43 (1972) 4536-42.

[13] Davies, D. K. and Biondi, M. A., J. Appl. Phys. 39 (1968) 2879-90.
[14] Latham, R. V. and Braun, E., J. Phys. D. 3 (1970) 1663-69.

[15] Boulloud, A., Le Vide, no 99 (1962) 240-3.

[16] RudolPH, V., Z. Naturforsch. 21a (1966) 1993-96.

[17] Rudolph, V., Z. Naturforsch. $24 a$ (1969) 326-31.

[18] Shelton, H., Hendricks, C. D., Wuerker, R. F., J. Appl. Phys. 31 (1960) 1243-46.

[19] Fritchtenicht, J. F., Rev. Sci. Instrum. 33 (1962) 209-12.

[20] Texier, C., Thèse de $3^{\mathrm{e}}$ cycle, Université de Nantes, 1973.

[21] Hertz, H., J. Reine Angew. Math. 92 (1886) 156-71.

[22] Andrews, J. P., Phil. Mag. S 79 (1930) 593-610.

[23] Taвor, D., Proc. Roy. Soc. A 192 (1948) 247-74.

[24] Martel, A., Comm. des Méthodes d'Essais des matériaux de construction (1895).

[25] Prescott, J., Appl. Elastic. (1927) London.

[26] CooK, M. A., « The Science of high Explosives » (Reinhold Edition, New-York) 1958.

[27] Bowden, F. P. and TABOR, D., The Friction and Lubrification of Solids (Oxford-Clarendon Press) T. 1, 1964. 